\title{
Development of an Intelligent Maximum Power Point Tracker Using an Advanced PV System Test Platform
}

\author{
Sergiu Spataru, Anastasios Amoiridis, Remus N. Beres, Catalin-Iosif Ciontea, Théo Klein, and Dezso Sera \\ Aalborg University, Aalborg, 9220, Denmark
}

\begin{abstract}
The performance of photovoltaic systems is often reduced by the presence of partial shadows. The system efficiency and availability can be improved by a maximum power point tracking algorithm that is able to detect partial shading conditions and to optimize the power output. This work proposes an intelligent maximum power point tracking method that monitors the maximum power point voltage and triggers a current-voltage sweep only when a partial shadow is detected, therefore minimizing power loss due to repeated current-voltage sweeps.

The proposed system is validated on an advanced, flexible photovoltaic inverter system test platform that is able to reproduce realistic partial shadow conditions, both in simulation and on hardware test system.
\end{abstract}

Index Terms - diagnostics, fault detection, maximum power point tracking, partial shading, photovoltaic systems.

\section{INTRODUCTION}

Partial shading (PS) is a common reason of power loss in a photovoltaic (PV) system due to irradiation reduction and mismatch, especially in case of residential applications [1, 2]. It is a well-known fact that partial shadows can create power loss that is over-proportional to the shadowed area. Depending on the relative position of the shadow with the bypass diodes configuration, even a small area shadow can cause very high power losses [2].

These losses can be further aggravated by the creation of multiple maximum power points (MPPs) on the array's power-voltage $(\mathrm{P}-\mathrm{V})$ curve. The common maximum power point tracking (MPPT) methods such as perturb and observe (P\&O) [3] or incremental conductance (INC) [4] have local maxima behavior, meaning that if a local maxima on the $\mathrm{P}-\mathrm{V}$ curve is found, it will stop there [5].

Several Global MPPT methods for solving this problem have been previously proposed, such as the a two stage global MPPT method presented in [6], the Global Peak (GP) MPPT proposed in [7], or the Real MPPT detailed in [8]. These methods are characterized by different degrees of complexity and applicability and are often based on periodically changing of the MPPT operation point, open-circuit voltage and shortcircuit current measurements $[6,8]$, or a complex model/MPPT algorithm [7].

A simple solution to this problem is to scan periodically the current-voltage (I-V) characteristic curve of the PV generator, in order to track the global MPP. However, this process causes energy loss, since during the scanning process the operating point is away from the MPP [5]. Methods have been presented with the aim of optimizing the scanning process, e.g. in function of the time of day [9].

Considering these aspects, the present paper proposes an intelligent MPPT (iMPPT) method for detecting PS conditions, finding the global MPPT, and thus increasing the energy yield of the system when operation in PS conditions.

The proposed method is based on I-V curve scanning, which is triggered asynchronously by a partial shadow detection unit, in order to minimize power loss due to unnecessary $\mathrm{I}-\mathrm{V}$ scanning.

The novelty of the method is represented by the partial shadow detection unit, which monitors the normal operation band/zone of voltage of the MPPT continuously, and is able to detect when the MPPT voltage is perturbed by the presence of PS. Once the PS has been detected, an I-V scan is triggered, the global MPP is calculated and the MPPT operation point is changed accordingly.

One important advantage of the iMPPT method is its simplicity, since the partial shadow detection unit can be combined with any MPPT algorithm, such as $\mathrm{P} \& \mathrm{O}$, as is the case in the present paper.

The sensitivity and robustness of the PS detection can be improved by compensating the natural perturbations in MPPT voltage, generated by changes in irradiance and module temperature, if ambient sensor measurements are available.

In case of multiple peaks, the proposed iMPPT method can substantially increase the energy yield from the PV system, especially if common MPPT methods track the local MPP. To illustrate the full advantages and benefits, the experimental validation of the method use real array data for multiple days under certain PS conditions. Moreover, the developing and testing process is applied on a high flexibility PV system test platform, using experimentally measured I-V curves, from PV arrays affected by partial shadows.

This paper is structured as follows. In section II the basic principle of the proposed MPPT method as well as the coordination of the MPPT with the PS unit are presented. Section III describes the development of the flexible PV system test platform used to test the proposed iMPPT system. Once both the proposed method and the test platform are described, the experimental tests and results are presented in section IV, followed by the conclusions.

\section{PRoposed INTELLIGENT MPPT CONCEPT}

The objective of the MPPT is to extract the maximum possible power from the PV system in all environmental 
conditions, including partial shading. In order to avoid the continuous I-V scanning, this work proposes a system based on a simple partial shadow detection method, that will trigger I-V scan only when PS is detected, called iMPPT.

The flowchart of the iMPPT concept is shown in Fig. 1, below. It is divided in two main parts: the partial shadow detection unit and the global MPPT unit.

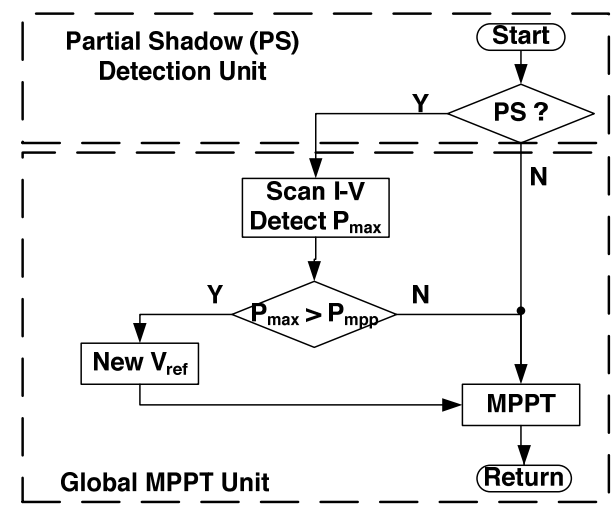

Fig. 1 iMPPT concept resulted from the cooperation partial shadow detection unit, an I-V scan procedure and of a regular MPPT (P\&O).

\section{A. Partial Shading Detection Unit}

In previous work [10] it was found that the thermal voltage, calculated as in (1), can indicate the presence of certain types of partial shadows.

$$
V_{t}=\frac{\left(I_{s c}-I_{m p p}\right) V_{m p p}}{I_{m p p}}
$$

In order to find $V_{t}$ during operation, the short-circuit current $\left(I_{s c}\right)$ is required to be calculated or measured; all other parameters involved in the equation of $V_{t}$ are given by the MPPT in steady state. In [11] is shown that $I_{s c}$ can be calculated as function of the MPP current $\left(I_{m p p}\right)$ over a wide range of irradiances (350-1000 $\mathrm{W} / \mathrm{m}^{2}$ ) using (2). In this way $I_{s c}$ can be estimated during the MPPT operation.

$$
K=\frac{I_{m p p}}{I_{s c}}
$$

However, assuming $\mathrm{K}$ constant in all operating conditions, the thermal voltage can be rewritten as (3).

$$
V_{t}^{\prime}=\frac{(1-K) V_{m p p}}{K}
$$

Where $V_{t}^{\prime}$ denotes the thermal voltage calculated based on $\mathrm{K}$. If $V_{t}^{\prime}$ is referred to a base reference measurement ( 1 p.u.), then the thermal voltage at given condition becomes as in (4):

$$
V_{t_{-} p u}^{\prime}=\frac{V_{t}^{\prime}}{V_{t_{-} \text {base }}}=\frac{\frac{1-K}{K} V_{\text {mpp }}}{\frac{1-K}{K} V_{\text {mpp_base }}}=\frac{V_{\text {mpp }}}{V_{\text {mpp_base }}}=V_{\text {mpp } \_p u}
$$

Where $V_{m p p}$ represent the actual measured MPP voltage and $V_{\text {mpp_base }}$ denotes the base reference MPP voltage. From (4) it results that it is possible to detect the partial shadings by looking on the MPP voltage and comparing to a base reference value taken in non-PS conditions, or from the PV module datasheet.

In order to underline the effect PS can have on the P-V curve and on the $V_{m p p \_p u}$ parameter, four P-V curves of a PV string (from which three of them affected by partial shadows), are presented in Fig. 2.

The curves, measured close to Standard Testing Conditions (STC - $1000 \mathrm{~W} / \mathrm{m}^{2}, 25^{\circ} \mathrm{C}$ and air mass 1.5 ), show that the $V_{\text {mp_pu }}$ parameter is sensitive to the presence of PS, if the shadows are significant, and have the potential to cause multiple MPPs.

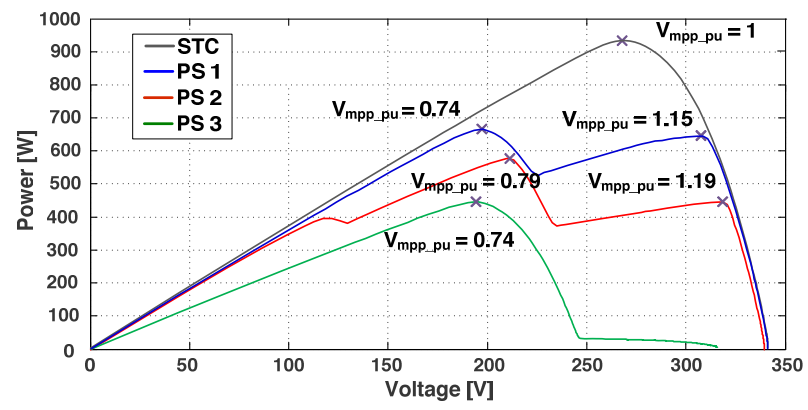

Fig. 2 Power-voltage characteristic of the PV string in normal operation and affected by three different partial shadows.

\section{B. Compensation for Ambient Conditions Variation}

During normal operation the $V_{m p p}$ varies with the change in module temperature and irradiance [12]. To consider the influence of ambient conditions, the actual value of $V_{m p p}$ can be translated to STC, using (5) [12].

$V_{\text {mpp }}=V_{\text {mpt }} p_{\text {meas }}-M_{s}\left[N_{s} V_{t} T_{c} \ln \left(\frac{G}{1000}\right)-\beta_{V m p p}\left(T_{c}-T_{S T C}\right)\right]$ (5)

Where: $M_{S}$ is the number of PV modules in series; $N_{S}$ is the number of series connected cells in a PV module; $V_{t}$ is the thermal voltage calculated as $A \cdot k \cdot T_{c} / q_{e}$ in which $A$ is diode ideality factor; $k$ is Boltzmann constant; $T_{c}$ is cell temperature, $q_{e}$ is charge of an electron, $\beta_{V m p p}$ is the temperature coefficient of the $V_{m p p}$.

Since the correction of $V_{m p p \_p u}$ to STC using (5) is not perfect, it is necessary to determine a normal operation band of the $V_{m p p}$ reference parameter.

In order to determine the limits for the normal operation band of the MPP voltage in different ambient/operation conditions, I-V characteristic curves of a PV array that consist of eight series BPMSX120 PV panels, were measured every 10 minutes for a duration of 5 days (during which time the PV array was not affected by any type of PS). The I-V curves were measured together with the POA irradiance and PV module temperature, with a Danfoss TLX Pro inverter.

In Fig. 3, the maximum power point voltage extracted from the I-V curves, translated to STC, and scaled to per-unit, is presented. The irradiance axis and module temperature scale have the purpose here to show the actual values of irradiance and temperature for each $V_{m p p}$ measurement. As can be 
observed, the translation to STC was not perfect, mainly due to inaccuracies in the translation equation parameters (only PV module datasheet parameters were used) and measurement errors from the Si irradiance sensor, which has an accuracy of $\pm 5 \%$. Even so a very narrow operation band of 0.945 p.u. to 1.055 p.u. has been found for the $V_{m p p}$ pu , parameter.

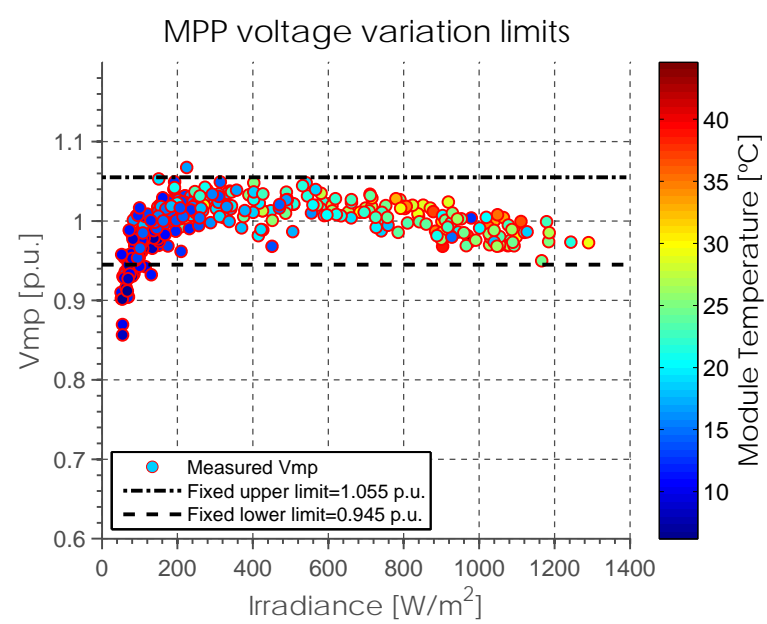

Fig. 3 Normal operation zone of the MPP voltage (50 and 1000 $\mathrm{W} / \mathrm{m}^{2}$ and $0{ }^{\circ} \mathrm{C}$ to $50^{\circ} \mathrm{C}$ ). The MPP voltage is translated to STC using irradiance and module temperature measurements. MPP voltage values outside these limits are considered to be caused by partial shadows.

This solution is found to work well for irradiances above $200 \mathrm{~W} / \mathrm{m}^{2}$, as for low irradiances the limitations of the translation equations become more apparent.

If irradiance and module temperature sensors are not available, the PS detection method can still be applied. In this case the limits of the MPP reference should be increased in order to take into account mainly the influence of temperature.

\section{Global MPPT Unit}

The global MPPT is consists of the I-V scan procedure and a regular MPPT algorithm, such as P\&O. The global MPPT unit is enabled by the partial shadow detection unit. Once enabled, it scans the I-V curve, detects the global MPP and sets the new MPPT voltage reference, if necessary.

The I-V scan procedure can be optimized to reduce energy loss and power fluctuations, by performing only a partial I-V scan. In the first stage the I-V scan can be limited to restricted $\mathrm{PV}$ array voltage range ( 0.5 to 0.9 p.u.). Secondly the direction of the I-V scan can be decided dynamically, for example if the MPPT voltage is below the detection band, the scan starts toward higher voltages, and vice versa if $V_{m p p}$ is above band, the I-V scan will start towards the lower voltages.

\section{DeVelopment And Testing Platform}

In order to test the previously described iMPPT, a high flexibility PV system testing platform has been developed, based on PV array I-V curve and ambient conditions measurements. The development and testing platform, presented in Fig. 4 consists of four different functional modules, described as follows.

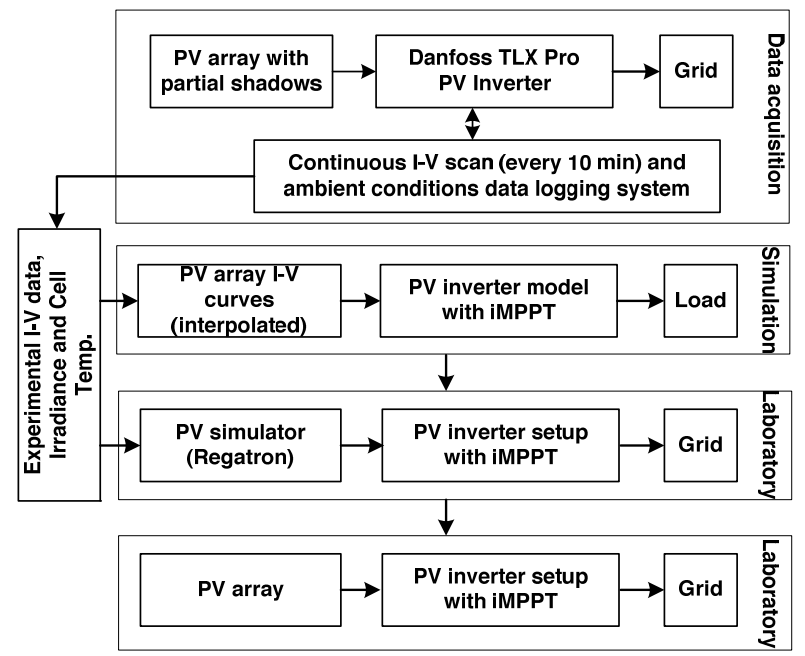

Fig. 4 Concept diagram of the development and testing platform. The same experimental I-V data is used in the simulations and laboratory tests.

\section{A. Photovoltaic array I-V data acquisition system}

The I-V characteristic curves of a PV array (two string of eight BPMSX120 mc-Si modules) is measured (every 10 minutes) with a Danfoss TLX Pro inverter. The I-V curves are logged together with the plane-of-array (POA) irradiance and PV module temperature, in a database.

Using this logging system, long term I-V monitoring data of the PV array is acquired with the aim of analyzing the field operation of the PV array, affected by different external factors such as partial shadows. This data is further used to develop partial shadow detection algorithms.

\section{B. $\quad$ iMPPT development and testing platform}

The initial development and testing cycle of the iMPPT control and partial detection algorithm is achieved using Matlab/Simulink. The iMPPT algorithm is implemented together with an average model of the PV inverter and grid as a Simulink model.

The input of the model is the experimental I-V curve data measured for the PV array every 10 minutes, together with the POI irradiance and module temperature. Since the I-V curves are acquired at discrete time intervals (10 minutes), and the dynamic of the simulation is much faster $(100 \mathrm{~Hz})$, the $\mathrm{I}-\mathrm{V}$ curves and ambient conditions are interpolated linearly between samples. Although in reality the irradiation does not vary linearly in the 10 minutes interval, the average irradiance can approximated to vary linearly within this interval, when considering larger time scales (such as days or weeks).

The advantage of this development and testing platform based on Matlab/Simulink, is that the operation and performance of the iMPPT control and partial shadow detection algorithm can be evaluated for several days or weeks 
in just a few minutes, while using real PV array I-V and ambient condition measurement data, acquired for those days/weeks.

\section{Experimental testing and validation platform}

For testing and validating the proposed iMPPT on a real hardware system, a $1 \mathrm{~kW}$ single-phase PV inverter testing platform (

Fig. 5) based on a Simulink/dSpace real-time system, was developed.

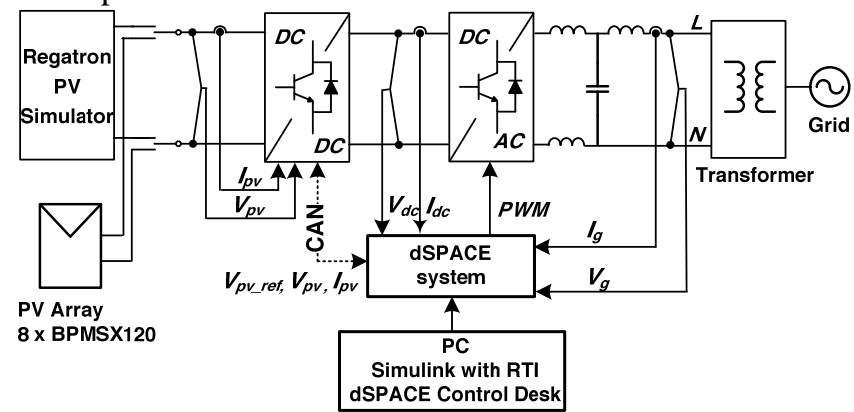

Fig. 5 Concept diagram of the PV experimental testing and validation platform, based on the dSpace/Simulink real-time control system.

The test setup consists of the following components: 1000V/40A high bandwidth PV simulator with a linear postprocessing unit, a custom-built 800W DC/DC boost converter connected to a $2.2 \mathrm{~kW}$ Danfoss VLT-FC302 inverter, which is then connected to the grid through an LC filter and a 1:1 transformer. The control structure has been implemented in Simulink and runs in real-time on the dSpace 1103 controller board. The DC/DC boost converter control and I-V scan procedure is implemented on a Texas Instruments TMS320F335 digital signal processor.

There is also the possibility to connect the PV inverter setup directly to a $0.8 \mathrm{kWp}$ PV string and perform long term testing and monitoring of the iMPPT operation.

\section{EXPERIMENTAL TESTS AND RESUltS}

In order to illustrate the iMPPT operation in partial shading conditions, two study cases are presented next. The first study case attempts to analyze performance of the iMPPT for long term operation (days), using experimental I-V data of a PV array affected by partial shadows. The second study case presents the transient operation (seconds) of the iMPPT implemented on the PV inverter setup.

\section{A. Long term operation and evaluation of the iMPPT}

To assess the iMPPT, measurements of I-V curves are taken for consecutive five days with a sampling rate of 10 minutes. The first day represents a baseline of the experimental study, when no PS is present on the PV array, and the system is operating normally. On the remaining four days several cells of two modules of the PV array are covered in order to create some PS. In the following, for each day, the iMPPT is assessed individually, and finally the performance results are summarized in Table 1.
In the first day, both classical P\&O and iMPPT tracked the MPP voltage almost ideally as illustrated in Fig. 6. It is worth to mention that in all considered I-V curves the iMPPT was not triggered, thus unnecessary I-V scans were avoided.

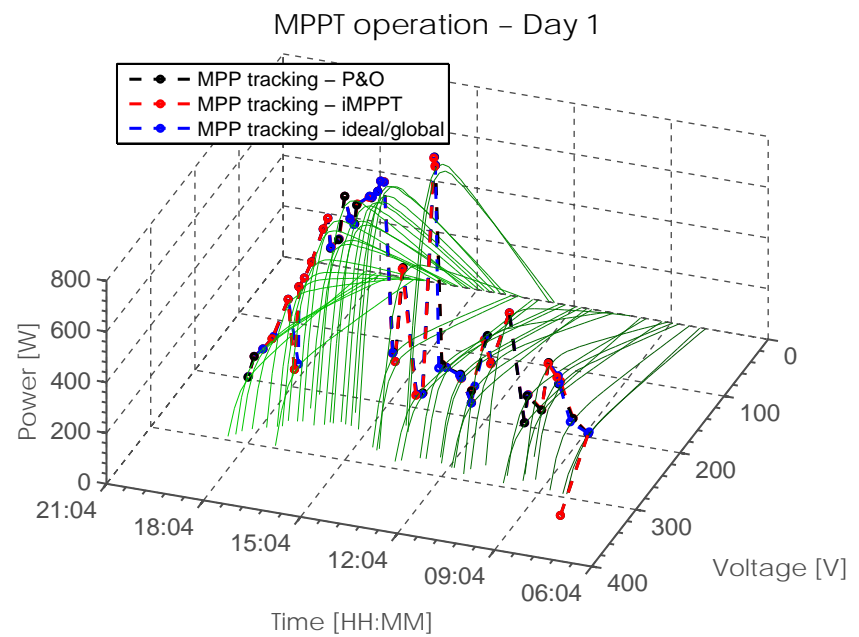

Fig. 6 P\&O, iMPPT and ideal MPPT operation profiles during day 1, when no partial shadows were present on the PV array, and both tracking algorithms operate near the ideal/global MPPT profile.

During day the, the behavior of both MPP trackers is similar until about 10:30am, when a PS starts to affect the PV array. Then P\&O losses tracking the global MPP and continue to track the local MPP. The iMPPT keeps tracking the global MPP all day. Comparison of both trackers with the ideal tracking of the global MPP is presented in Fig. 7.

In Fig. 8 the operation of both trackers is illustrated for the third day. Only in the morning for a limited amount of time there is a slight deviation from the global MPP of the P\&O while soon after both MPPTs are close to ideal tracking.

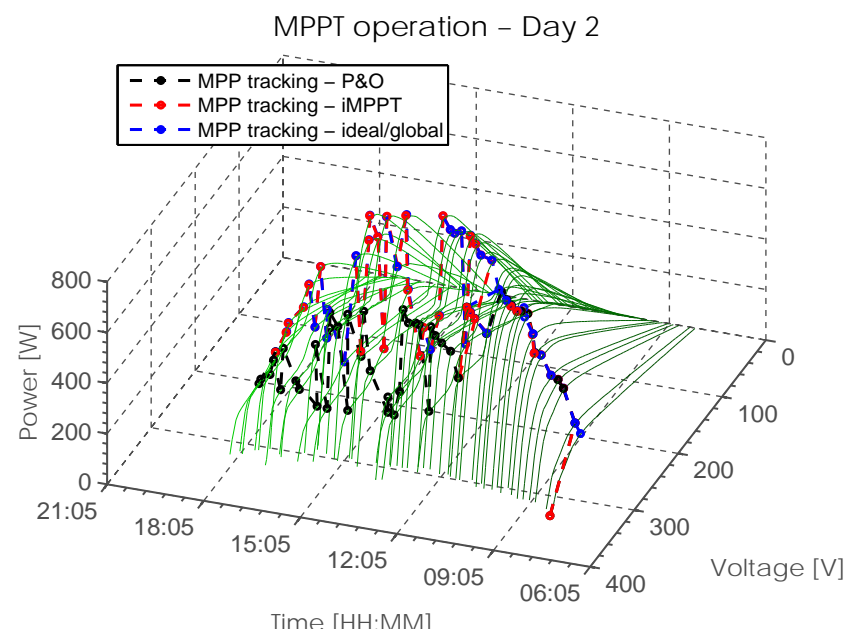

Fig. 7 P\&O, iMPPT and ideal MPPT operation profiles during day 2, when a partial shadow starts affecting the PV array in the morning. The P\&O losses the global MPP and operates on a local MPP for the rest of the day, whilst the iMPPT detects the partial shadow, scans the $\mathrm{I}-\mathrm{V}$ curve and adjust its operation near the global MPP. 


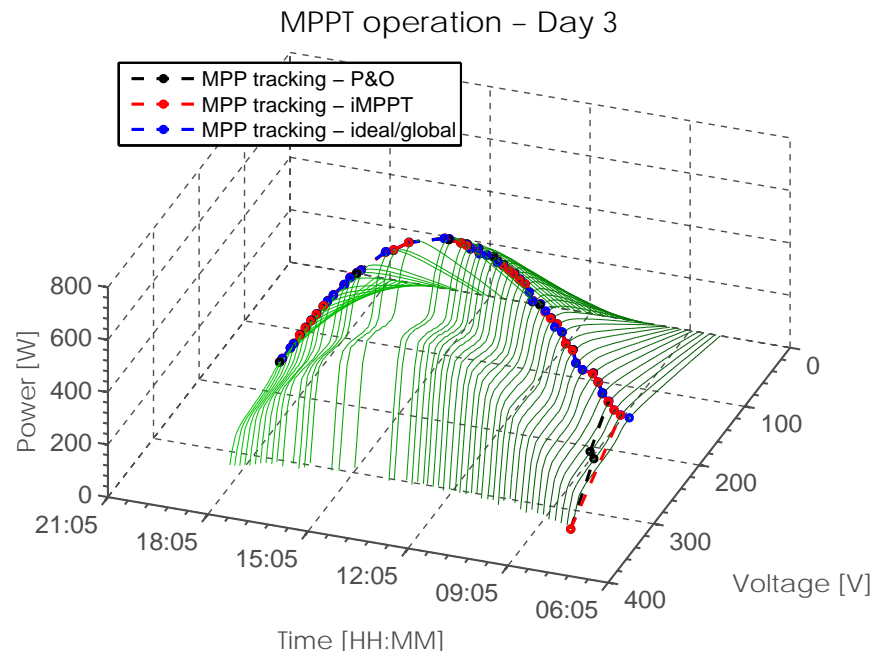

Fig. 8 P\&O, iMPPT and ideal MPPT operation profiles during day 3, when a partial shadow affects the PV array the entire day. Although a partial shadow affects the systems and multiple MPP are formed, both $\mathrm{P} \& \mathrm{O}$ and iMPPT operate near the ideal/global MPPT profile.

During day 4 (Fig. 9), the P\&O is stuck on the local MPP during inverter startup and remains at this point for the rest of the day. The iMPPT adjusts its operating point to the global MPP and keeps to track this point all day long.

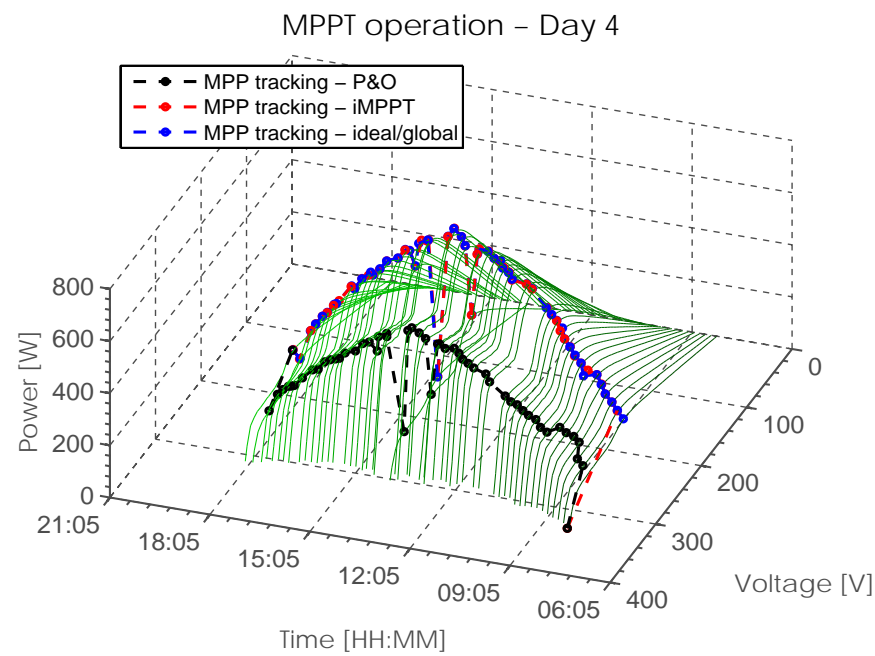

Fig. 9 P\&O, iMPPT and ideal MPPT operation profiles during day 4, when a partial shadow affects the PV array the entire day. The P\&O is stuck on the local MPP during inverter startup and remains at this point for the rest of the day. The iMPPT detects the partial shadow early on and is able to make the necessary adjustments to its MPP operation point.

During the last day, due to nature of PS, both P\&O and iMPPT trackers are able to find the global MPP when the inverter starts and remain near the ideal/global MPPT for the remainder of the day, as can be seen from Fig. 10.

From the study case it can be concluded that if the PV array is affected by PS, the P\&O may remain stuck at the local MPP. In this case some energy production is lost (depending on the severity of the PS), that can accumulate to a significant amount, for long term operation. In contrast, the iMPPT is triggered by the PS detection unit and thus is able to work only on the global MPP.

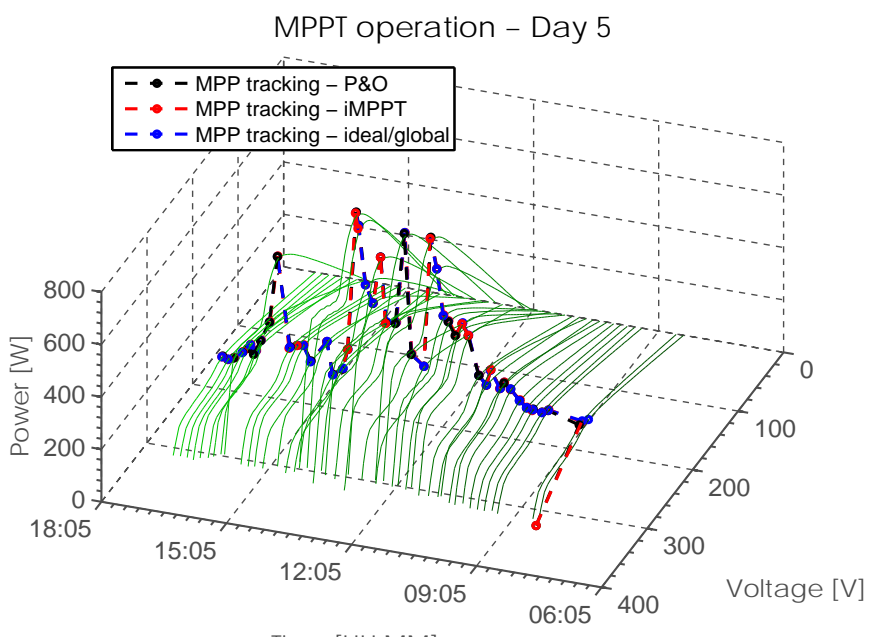

Time $[\mathrm{HH}: \mathrm{MM}]$

Fig. $10 \mathrm{P} \& \mathrm{O}$, iMPPT and ideal MPPT operation profiles during day 5, when a partial shadow affects the PV array the entire day.

Although a partial shadow is affecting the PV array, both the P\&O and iMPPT are able to find the global MPP when the inverter starts and remain near the ideal/global MPPT for the rest of the day.

In Table 1 the efficiencies of both MPP trackers are summarized, where the efficiency is calculated as the ratio between the actual energy yield during the considered day and the maximum possible energy yield from the array.

Table 1: P\&O/iMPPT efficiency

\begin{tabular}{|c|c|c|c|c|c|}
\hline & Day 1 & Day 2 & Day 3 & Day 4 & Day 5 \\
\hline P\&O & $99.9 \%$ & $84.6 \%$ & $99.5 \%$ & $75 \%$ & $99.87 \%$ \\
\hline iMPPT & $99.9 \%$ & $99.4 \%$ & $99.9 \%$ & $99.9 \%$ & $99.87 \%$ \\
\hline $\begin{array}{c}\text { Partial shadow } \\
\text { present? }\end{array}$ & No & Yes & Yes & Yes & Yes \\
\hline
\end{tabular}

\section{B. Transient operation and evaluation of the iMPPT}

To illustrate the practical implementation of the iMPPT, on the experimental testing and validation platform, a study case with the transient operation of the iMPPT is presented next.

The test contains a transition between a normal (un-shaded) I-V curve and the PS2 I-V curve from Fig. 2. Both I-V curves are stored in the Regatron PV simulator and switched at 1.3s as illustrated in Fig. 11. Because the PV array has an output power of $960 \mathrm{Wp}$ the un-shaded curve corresponds to an irradiance of $750 \mathrm{~W} / \mathrm{m}^{2}$ in order to obtain an output power lower than the dc-dc converter rated power.

The PS detection unit has three independent states: state 0 when the PS unit is disabled; state 1 when PS detection unit is enabled and $V_{\text {mpp }} p u$ is calculated only when array voltage reaches the MPP; state 2 correspond to detection of PS if the MPP voltage is outside the normal operation band, as defined in Fig. 3. However, as can be seen in Fig. 11, the trigger signal for the sweep function is sent only when the PS is detected 
first, at 3.2s. The sweep function will not be triggered after (at 4s) as MPP voltage is still outside the boundaries.
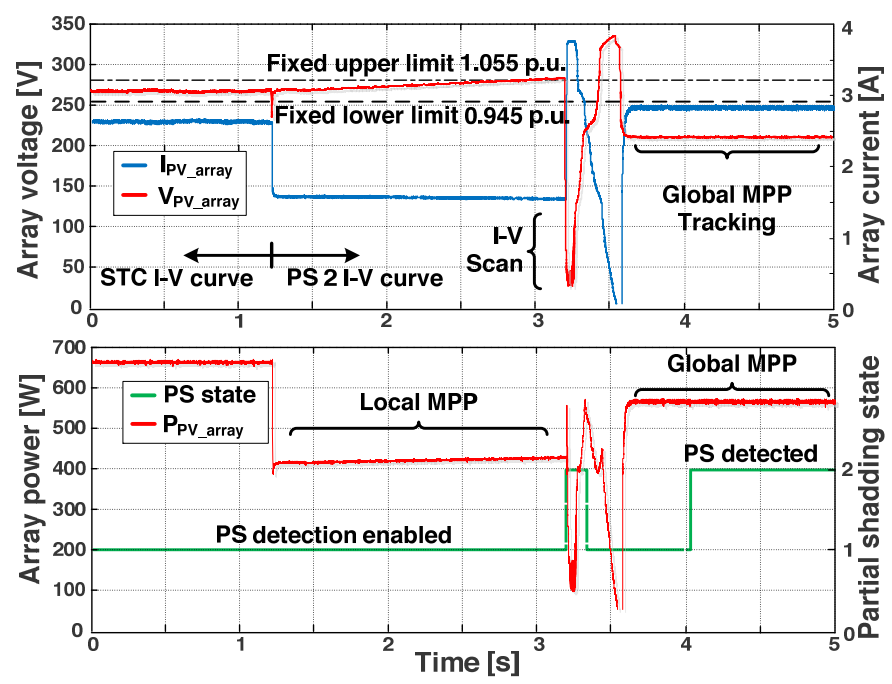

Fig. 11 Results of the partial shadowing detection test. The normal operation corresponds to the reference I-V curve in Fig.2, while the partial shadow condition corresponds to PS 2 from Fig 2.

The sudden appearance of a partial shadow is at some extent unrealistic since normally the shadows from fixed objects appear gradually, as the Sun moves on the sky. However, since the slow movement of the MPP voltage due to the moving shadow is perceived by the much faster MPPT as steady state, the PS detection is continuously enabled; therefore as soon as the MPP voltage moves out of the normal operation boundaries, the PS is detected.

The PS detection unit based on MPP voltage comparison has been proved to be effective in both simulations using real $\mathrm{I}-\mathrm{V}$ measured data and in experimental tests.

\section{CONCLUSIONS}

The proposed iMPPT concept combines the advantages of the I-V scan based MPPT - in that it always finds the global MPP - with the simple partial shadow detection unit. This minimizes the power losses due to the scanning process by activating the scan only when partial shadow conditions are detected.

The novelty of the method is represented by the partial shadow detection unit, which monitors the normal operation band/zone of voltage of the MPPT continuously, and is able to detect when the MPPT voltage is perturbed by the presence of PS. Once the PS has been detected, an I-V scan is triggered, the global MPP is calculated and the MPPT operation point is changed accordingly.

Two essential advantages of the method are its simplicity, since the partial shadow detection unit can be combined with any MPPT algorithm, such as $\mathrm{P} \& \mathrm{O}$, and its potential to improve the system performance in partial shadow conditions, with a minimal cost. Irradiance and module temperature sensors can improve the sensitivity and robustness of the method, but are not mandatory.

In order to test this method, a high flexibility PV system testing platform that uses real data measurements of different partial shadow conditions has been developed.

Simulation and experimental results showed that the global MPP is tracked accurately in all considered cases of partial shadows.

\section{REFERENCES}

[1] A. Woyte, J. Nijs, and R. Belmans, "Partial shadowing of photovoltaic arrays with different system configurations: literature review and field test results," Solar Energy, vol. 74, no. 3, pp. 217-233, 2003.

[2] B. Decker, and U. Jahn, "Performance of 170 grid connected PV plants in Northern Germany-Analysis of yields and optimization potentials," Solar Energy, vol. 59, no. 4-6, pp. 127133, 4//, 1997.

[3] D. P. Hohm, and M. E. Ropp, "Comparative study of Maximum Power Point Tracking algorithms using an experimental, programmable, maximum power point tracking test bed," Conference Record of the Twenty-Eighth IEEE Photovoltaic Specialists Conference - 2000, IEEE Photovoltaic Specialists Conference, pp. 1699-1702, New York: IEEE, 2000.

[4] K. H. Hussein, I. Muta, T. Hoshino, and M. Osakada, "Maximum Photovoltaic Power Tracking - an Algorithm for Rapidly Changing Atmospheric Conditions," IEE ProceedingsGeneration Transmission and Distribution, vol. 142, no. 1, pp. 59-64, Jan, 1995.

[5] T. Esram, and P. L. Chapman, "Comparison of photovoltaic array maximum power point tracking techniques," IEEE Transactions on Energy Conversion, vol. 22, no. 2, pp. 439-449, Jun, 2007.

[6] K. Kobayashi, I. Takano, and Y. Sawada, “A study of a two stage maximum power point tracking control of a photovoltaic system under partially shaded insolation conditions," Solar Energy Materials and Solar Cells, vol. 90, no. 18-19, pp. 29752988, 11/23/, 2006.

[7] H. Patel, and V. Agarwal, "Maximum Power Point Tracking Scheme for PV Systems Operating Under Partially Shaded Conditions,” Industrial Electronics, IEEE Transactions on, vol. 55, no. 4, pp. 1689-1698, 2008.

[8] J. Young-Hyok, J. Doo-Yong, K. Jun-Gu, K. Jae-Hyung, L. TaeWon, and W. Chung-Yuen, "A Real Maximum Power Point Tracking Method for Mismatching Compensation in PV Array Under Partially Shaded Conditions,” Power Electronics, IEEE Transactions on, vol. 26, no. 4, pp. 1001-1009, 2011.

[9] S. B. Kjær, O. Oprea, and U. Borup, "Adaptive Sweep for PV Applications," in 26th European Photovoltaic Solar Energy Conference and Exhibition, Hamburg, Germany, 2011, pp. 3708 - 3710.

[10] D. Sera, "Real-time Modelling, Diagnostics and Optimised MPPT for Residental PV Systems,” Department of Energy Technology, Aalborg University, Aalborg, Denmark, 2009.

[11] D. Sera, L. Mathe, T. Kerekes, R. Teodorescu, and P. Rodriguez, "A low-disturbance diagnostic function integrated in the PV arrays' MPPT algorithm,” in IECON 2011 - 37th Annual Conference on IEEE Industrial Electronics Society, Melbourne, Australia, 2011, pp. 2456-2460.

[12] D. L. King, W. E. Boyson, and J. A. Kratochvil, Photovoltaic Array Performance Model, Sandia National Laboratories, Albuquerque, New Mexico 87185-0752, 2004. 